\title{
From standard wind measurements to spectral characterization: turbulence length scale and distribution
}

\author{
Mark Kelly \\ Wind Energy Department, Risø Lab./Campus, Danish Technical University, Roskilde 4000, Denmark \\ Correspondence: Mark Kelly (mkel@dtu.dk)
}

Received: 11 February 2018 - Discussion started: 14 February 2018

Revised: 3 July 2018 - Accepted: 24 July 2018 - Published: 16 August 2018

\begin{abstract}
In wind energy, the effect of turbulence upon turbines is typically simulated using wind "input" time series based on turbulence spectra. The velocity components' spectra are characterized by the amplitude of turbulent fluctuations, as well as the length scale corresponding to the dominant eddies. Following the IEC standard, turbine load calculations commonly involve use of the Mann spectral-tensor model to generate time series of the turbulent three-dimensional velocity field. In practice, this spectral-tensor model is employed by adjusting its three parameters: the dominant turbulence length scale $L_{\mathrm{MM}}$ (peak length scale of an undistorted isotropic velocity spectrum), the rate of dissipation of turbulent kinetic energy $\varepsilon$, and the turbulent eddy-lifetime (anisotropy) parameter $\Gamma$. Deviation from "ideal" neutral sheared turbulence - i.e., for non-zero heat flux and/or heights above the surface layer - is, in effect, captured by setting these parameters according to observations.

Previously, site-specific $\left\{L_{\mathrm{MM}}, \varepsilon, \Gamma\right\}$ values were obtainable through fits to measured three-dimensional velocity component spectra recorded with sample rates resolving the inertial range of turbulence $(\gtrsim 1 \mathrm{~Hz})$; however, this is not feasible in most industrial wind energy projects, which lack multi-dimensional sonic anemometers and employ loggers that record measurements averaged over intervals of minutes. Here a form is derived for the shear dependence implied by the eddy-lifetime prescription within the Mann spectral-tensor model, which leads to derivation of useful forms of the turbulence length scale. Subsequently it is shown how $L_{\mathrm{MM}}$ can be calculated from commonly measured site-specific atmospheric parameters, namely mean wind shear $(\mathrm{d} U / \mathrm{d} z)$ and standard deviation of streamwise fluctuations $\left(\sigma_{u}\right)$. The derived $L_{\mathrm{MM}}$ can be obtained from standard (10 min average) cup anemometer measurements, in contrast with an earlier form based on friction velocity.

The new form is tested across several different conditions and sites, and it is found to be more robust and accurate than estimates relying on friction velocity observations. Assumptions behind the derivations are also tested, giving new insight into rapid-distortion theory and eddy-lifetime modeling - and application - within the atmospheric boundary layer. The work herein further shows that distributions of turbulence length scale, obtained using the new form with typical measurements, compare well with distributions $P\left(L_{\mathrm{MM}}\right)$ obtained by fitting to spectra from research-grade sonic anemometer measurements for the various flow regimes and sites analyzed. The new form is thus motivated by and amenable to site-specific probabilistic loads characterization.
\end{abstract}




\section{Introduction}

Of the atmospheric parameters which are generally input into (or required by) wind turbine load calculation codes, several stand out due to their prominence in load contributions: the "mean" wind speed $U$, the standard deviation of streamwise turbulent velocity $\sigma_{u}$, the shear $\mathrm{d} U / \mathrm{d} z$ or shear exponent $\alpha$, and the characteristic turbulence length scale $L$ corresponding to the most energetic turbulent motions (e.g., Wyngaard, 2010). Dimitrov et al. (2015) explored the importance of shear $(\alpha)$; Dimitrov et al. (2017) found that both fatigue and extreme turbine loads can be sensitive to $L$ in addition to the dominant influences of mean wind speed $U$ and streamwise turbulence "strength" $\sigma_{u}{ }^{1}$. These are also consistent with the earlier finding of Sathe et al. (2013) that stability could affect fatigue loads through $\alpha$ and $\sigma_{u}$.

Within the context of obtaining site-dependent statistics of the most crucial load-driving parameters $\left(U, \sigma_{u}, \alpha, L\right)$ from conventional industrial wind measurements, this work focuses on the one parameter which has thus far been most difficult to measure: the turbulence length scale $L$. The turbulence length scale corresponds to the "energy-containing sub-range" of turbulent velocity fluctuations associated with the peak of the streamwise velocity spectrum, which contribute most to turbulent kinetic energy (and $\sigma_{u}$ ) - and which can dominate the turbulence contribution to wind turbine loads. Measurements used in wind energy are usually stored as $10 \mathrm{~min}$ statistics (average and standard deviation of wind speed and direction), so one cannot obtain turbulence spectra from them, nor can one calculate integral time or length scales from such observations.

Because of its widespread use in the wind industry and its inclusion in the IEC 61400-1, Edition 3 (2005) standard on design requirements for wind turbines, here we consider the spectral turbulence model of Mann (1994) and $L$ as prescribed for this model. Within the "Mann model", which uses rapid-distortion theory (RDT) to account for shear-induced distortion of isotropic turbulence (e.g., Savill, 1987; Pope, 2000), there is also a prescription for the scale-dependent time over which turbulent eddies of a given size are distorted. This timescale is key to proper representation of atmospheric turbulence and reproduction of component spectra via RDT. However, the eddy lifetime was not directly derived, but rather cleverly prescribed, by Mann (1994). Concurrent to and independent of the work herein, de Mare and Mann (2016) also derived some relations to create a model for time-varying eddy lifetime. The present article provides direct derivation of the eddy lifetime, which results in a relation between the three (spectral) parameters of the Mann model and measurable quantities. More importantly, the derivations here include connection of the turbulence length scale to routinely available quantities from typical $10 \mathrm{~min}$ industrial

\footnotetext{
${ }^{1}$ To a lesser extent, some sensitivity to the Mann-model anisotropy parameter $\Gamma$ has also been found.
}

wind records. The turbulence length scale is in fact that corresponding to the von Kármán (1948) spectral form, and thus the relation here is applicable to other turbulence models used in wind engineering, such as those relying on the Kaimal et al. (1972) spectrum.

After deriving the eddy lifetime and giving subsequent expressions for the turbulence length scale, this article proceeds to validation of the underlying assumptions. Constraints implied by fitting the Mann model to measured spectra in nonneutral conditions, given eddy lifetime and mixing-length relations, are also tested. This includes dependence of predicted velocity variance on model anisotropy parameter $(\Gamma)$, as well as implications in the surface layer and connection to previous findings in boundary-layer meteorology. Finally, the length scale obtained from conventional $10 \mathrm{~min}$ wind measurements via the new expression is compared to the length scale found from fits of Mann-model output to measured component spectra; this is done using data from multiple sites, representing several types of site conditions.

\section{Theory}

Relation of the turbulence length (spectral "peak") scale to measurable statistics is possible through the eddy-lifetime form of Mann (1994), where the latter is defined in terms of the isotropic von Kármán spectrum that is distorted using RDT.

\subsection{Eddy lifetime}

A number of forms exist to estimate eddy lifetime $\tau_{\mathrm{e}}$, though these can be generally expressed as the ratio of a length scale (taken as the reciprocal of wavenumber, $k^{-1}$ ) to a velocity scale which follows from some integrated form of the (scalar) kinetic energy spectrum $E(k)$ :

$\tau_{\mathrm{e}} \sim k^{-p-1}\left[\int_{k}^{\infty} \kappa^{-2 p} E(\kappa) \mathrm{d} \kappa\right]^{-1 / 2}$,

where the characteristic velocity scale can be generically described by

$k^{p}\left[\int_{k}^{\infty} \kappa^{-2 p} E(\kappa) \mathrm{d} \kappa\right]^{1 / 2}$.

In contrast to the "coherence-destroying diffusion time" of Comte-Bellot and Corrsin (1971) and the reciprocal of eddydamping rates from Lesieur (1990), for use with rapiddistortion theory Mann (1994) chose an eddy lifetime that depends on eddy size (wavenumber) according to

$\tau_{\mathrm{M}} \propto k^{-1}\left[\int_{k}^{\infty} E(\kappa) \mathrm{d} \kappa\right]^{-1 / 2}$, 
i.e., equivalent to $p=0$ in terms of Eq. (1). The choice of Eq. (2) for eddy lifetime was found to behave more reasonably than both the Comte-Bellot and Corrsin (1971) "diffusion time" (where $p=1)^{2}$, as well as the timescale $\left[k^{3} E(k)\right]^{-1 / 2}$ (which in the inertial range is equivalent to $p=-1)^{3}$ implicit in eddy-damped quasi-normal Markovian models (Andre and Lesieur, 1977; Lesieur, 1990); both of the latter lifetime models do not (reliably) integrate to give finite $\sigma_{u}^{2}$.

Mann (1994) re-writes $\tau_{M}$ as

$$
\tau_{\mathrm{M}}(k)=\frac{\Gamma}{\mathrm{d} U / \mathrm{d} z} \frac{\left(k L_{\mathrm{MM}}\right)^{-2 / 3}}{\sqrt{2 F_{1}\left(\frac{1}{3}, \frac{17}{6} ; \frac{4}{3} ; \frac{-1}{\left(k L_{\mathrm{MM}}\right)^{2}}\right)}},
$$

where ${ }_{2} F_{1}$ is Gauss' hypergeometric function (Abramowitz and Stegun, 1972) ${ }^{4}$, and $L_{\mathrm{MM}}$ is the turbulence length scale associated with the peak of the turbulent kinetic energy spectrum $E(k)$ as in Eq. (2). The eddy lifetime definition (3) is used in practical implementation of the spectral tensor model (e.g., Mann, 2000), and it notably defines a parameter of this model: the eddy lifetime factor $\Gamma$, also known as the anisotropy factor. The Mann (1994) spectral-tensor model employs RDT, whereby the shear $\mathrm{d} U / \mathrm{d} z$ distorts turbulence from an isotropic state, based on an initial turbulent kinetic energy spectrum of the von Kármán form

$$
E_{\mathrm{vK}}(k)=\alpha \varepsilon^{2 / 3} L_{\mathrm{MM}}^{5 / 3} \frac{\left(k L_{\mathrm{MM}}\right)^{4}}{\left[1+\left(k L_{\mathrm{MM}}\right)^{2}\right]^{17 / 6}},
$$

where $\alpha=1.7$ (von Kármán, 1948). This in effect defines the length scale $L_{\mathrm{MM}}$ through the peak of the initial spectrum ${ }^{5}$. Using Eq. (4) in the proportionality expression (2) produces

$$
\tau_{\left.M\right|_{E \rightarrow E_{\mathrm{VK}}}}=\frac{c_{\tau} k^{-2 / 3}}{\sqrt{\frac{3}{2} \alpha \varepsilon_{2}^{2 / 3} F_{1}\left(\frac{1}{3}, \frac{17}{6} ; \frac{4}{3} ; \frac{-1}{\left(k L_{\mathrm{MM})^{2}}\right.}\right)}},
$$

where we have introduced the proportionality constant $c_{\tau}$ to write the result of integrating the proportionality relation (2)

\footnotetext{
${ }^{2}$ The Mann (1994) expression is also equivalent (or at least proportional) to the "convection time" of Comte-Bellot and Corrsin (1971).

${ }^{3}$ The reciprocal of eddy-damping rate, $\left[k^{3} E(k)\right]^{-1 / 2}$, is equal in the inertial range to Eq. (1) with $p=-1$ since $E(\kappa) \rightarrow \kappa^{-5 / 3}$ there. This expression is also similar to the "rotation time" or "strain time" given by Comte-Bellot and Corrsin (1971), but it should be noted that such expressions integrate from 0 to $k$, i.e., over eddies larger than $1 / k$.

${ }^{4}$ The hypergeometric function ${ }_{2} F_{1}\left[\frac{1}{3}, \frac{17}{6} ; \frac{4}{3} ;-\left(k L_{\mathrm{MM}}\right)^{-2}\right]$ approaches 1 for $k L_{\mathrm{MM}} \gg 1$ (the inertial range) and simplifies to $a_{\mathrm{HG}}\left(k L_{\mathrm{MM}}\right)^{2 / 3}$ for $k L_{\mathrm{MM}} \ll 1$, where $a_{\mathrm{HG}} \equiv(3 \sqrt{\pi} / 4) f_{\Gamma}(4 / 3) / f_{\Gamma}(17 / 6) \simeq 0.69$ and $f_{\Gamma}(x)$ is the Euler gamma function.

${ }^{5}$ The peak of the von Kármán isotropic TKE spectrum $E_{\mathrm{vK}}(k)$ occurs at $k L_{\mathrm{MM}}=\sqrt{12 / 5}$, i.e., $L_{\mathrm{MM}} \simeq 1.55 / k_{\text {peak }}$.
}

as an equation. Now $\tau_{\mathrm{M}}$ can be seen to depend upon $k$, $L_{\mathrm{MM}}$, and $\varepsilon$. The eddy lifetime can be reduced and clarified via ${ }_{2} F_{1}\left\{\frac{1}{3}, \frac{17}{6} ; \frac{4}{3},\left(-k L_{\mathrm{MM}}\right)^{-2}\right\} \simeq\left[1+3.07\left(k L_{\mathrm{MM}}\right)^{-2}\right]^{-1 / 3}$ to give the more transparent von Kármán-like form ${ }^{6}$

$$
\tau_{\mathrm{M}}\left(k ; L_{\mathrm{MM}}, \varepsilon\right) \simeq \frac{0.82 c_{\tau}}{\sqrt{\alpha \varepsilon^{2 / 3}}} k^{-2 / 3}\left[1+\frac{3.07}{\left(k L_{\mathrm{MM}}\right)^{2}}\right]^{1 / 6} .
$$

Since Eqs. (3) and (5) are equal, we have an expression relating the Mann-model parameters to the shear $\mathrm{d} U / \mathrm{d} z$ :

$\Gamma=\frac{c_{\tau}}{\sqrt{3 \alpha / 2}} \frac{\mathrm{d} U}{\mathrm{~d} z} L_{\mathrm{MM}}^{2 / 3} \varepsilon^{-1 / 3}$.

The expression (7) can be made yet more useful to relate the turbulent length scale to measurable parameters, as shown in Sect. 2.2.

\section{Eddy lifetime and equilibrium}

The parameters $\left\{\varepsilon, \Gamma, L_{\mathrm{MM}}\right\}$ are site-dependent and in practice have been obtained from measurements through fits of the model output to observed spectra (Mann, 2000), relying on (at least three of) $F_{11}, F_{13}, F_{33}$, and $F_{22}$ (e.g., Sathe et al., 2013; Dimitrov et al., 2017). The model starts with an (undistorted) isotropic incompressible turbulence spectral tensor:

$\left.\Phi_{i j}(\boldsymbol{k})\right|_{0}=\frac{\delta_{i j} k^{2}-k_{i} k_{j}}{4 \pi k^{4}} E(k)$,

where $E(k)$ is taken to be $E_{\mathrm{vK}}(k)$ (shown in Eq. 4), then the $\Phi_{i j}$ are distorted - i.e., the rapid-distortion equations are solved - per (three-dimensional) wavenumber over a time $\tau_{\mathrm{M}}(k)$ via RDT.

The rapid-distortion equations do not explicitly solve for production of normal stresses (which sum to twice the turbulent kinetic energy) or shear stress, though they do include terms that perturb the stresses ${ }^{7}$ to account for the (anisotropic) effect of a constant shear $\mathrm{d} U / \mathrm{d} z$. Further, the RDT discussed here does not include dissipation (Mann, 1994; Pope, 2000); instead, in the spectral-tensor model the dissipation rate of turbulent kinetic energy $\varepsilon$ is a parameter giving the amplitude of the undistorted (initial) spectrum via Eq. (4). In practice $\varepsilon$ is obtained via fits of pre-calculated Mann-model output to measured spectra. So $\varepsilon$ in effect gives

\footnotetext{
${ }^{6}$ Note $\sqrt{2 / 3} \simeq 0.82$ and $3.07=a_{\mathrm{HG}}^{-3}$; cf. footnote 4. In Eq. (6), $\alpha \varepsilon^{2 / 3}$ is kept together for comparison with Eq. (4) and because $\alpha \varepsilon^{2 / 3}$ is commonly used as an input to the spectral-tensor model instead of $\varepsilon$ (e.g., Mann et al., 2002; IEC 61400-1, Edition 3, 2005).

${ }^{7}$ Assuming a constant mean shear $\mathrm{d} U / \mathrm{d} z$, the spectraltensor model solves Fourier-transformed versions of rapiddistortion equations for streamwise normal stress $\left\langle u_{1} u_{1}\right\rangle$ and shear stress $\left\langle u_{1} u_{3}\right\rangle$; multiplying these by $\mathrm{d} U / \mathrm{d} z$ one obtains the corresponding production rates: $P_{11}=-2\left\langle u_{1} u_{3}\right\rangle \mathrm{d} U / \mathrm{d} z$ and $P_{13}=-\left\langle u_{3} u_{3}\right\rangle \mathrm{d} U / \mathrm{d} z$ (Pope, 2000, chap. 11).
} 
the inertial-range amplitudes of the distorted velocity component spectra, which have been distorted for a time $\tau_{\mathrm{M}}(k)$. From Eq. (3) one sees that the parameter $\Gamma$ serves as a factor that determines the amount of distortion and associated anisotropy: increasing $\Gamma$ corresponds to longer distortion time $\tau_{\mathrm{M}}$ and thus more anisotropy, with $\Gamma=0$ corresponding to isotropy (zero distortion of the initial isotropic $\Phi_{i j}$ ). The separation between the peaks of the different component spectra increases with $\Gamma$; the spectral peak of $F_{33}$ is at higher wavenumbers (smaller scales) than the $F_{22}$ peak, which is at higher wavenumbers than the peak of $F_{11}$ (Mann, 1994).

A stationary equilibrium result is achieved via the eddylifetime prescription together with rapid distortion of the isotropic spectral tensor - with $\tau_{\mathrm{M}}$ and (initial) inertial-range amplitudes depending on $\varepsilon$ via Eqs. (3)-(4) and (7), whereby shear-production of TKE is in effect balanced by dissipation. That is, the resultant shear stress $\langle u w\rangle$ (expressible now in terms of $\varepsilon$ ) can be multiplied by $2 \partial U / \partial z$ to give the implied production rate of $\langle u u\rangle$, which with $v v$ and $w w$ (through $\Gamma$ ) gives the implied TKE production rate, amounting to $P=\varepsilon$; such an equilibrium, enforced by $\tau_{\mathrm{M}}$, can also be inferred from de Mare and Mann (2016).

\subsection{Characteristic length scale}

Noting that the spectrum of a variable integrates to the variance of said variable, then invoking Eq. (8) with the isotropic von Kármán form Eq. (4) for $E(k)$ and exploiting $F_{11}\left(k_{1}\right)=\iint \Phi_{11} \mathrm{~d} k_{2} \mathrm{~d} k_{3}$, one obtains the isotropic streamwise turbulence variance

$$
\begin{aligned}
\sigma_{\text {iso }}^{2} & =2 \int_{0}^{\infty} \frac{9}{55} \alpha \varepsilon^{2 / 3} L_{\mathrm{MM}}^{5 / 3}\left[1+\left(k_{1} L_{\mathrm{MM}}\right)^{2}\right]^{-5 / 6} \mathrm{~d} k_{1} \\
& =0.69 \alpha \varepsilon^{2 / 3} L_{\mathrm{MM}}^{2 / 3},
\end{aligned}
$$

which is the undistorted streamwise variance. The factor 0.69 is the numerical value of $\frac{9}{55} \sqrt{\pi} f_{\Gamma}\left(\frac{1}{3}\right) / f_{\Gamma}\left(\frac{5}{6}\right)$, and $f_{\Gamma}(x)$ is the Euler gamma function (Abramowitz and Stegun, 1972, see also footnote 4). Then using Eq. (9) in Eq. (7) we get a relation for the isotropic (undistorted) turbulence length scale implied by the lifetime model (3),

$$
L_{\mathrm{MM}} \simeq\left(\frac{1.5 \Gamma}{c_{\tau}}\right) \frac{\sigma_{\text {iso }}}{\mathrm{d} U / \mathrm{d} z},
$$

where the leading term in parentheses is expected to be of the order of 1.

\subsubsection{Relation to observations}

Peña et al. (2010) suggested that the Mann-model length scale is proportional to the classic mixing length $\ell_{*} \equiv u_{*} /(\mathrm{d} U / \mathrm{d} z)$ multiplied by an empirical constant,

$L_{\mathrm{MM}}=c_{m} \ell_{*}=\frac{c_{m} u_{*, \mathrm{obs}}}{\mathrm{d} U / \mathrm{d} z}$, where they assign $c_{m}=1.7$. However, we find from observations that on average $c_{m} \approx 2.3$ over flat land, i.e., $\left\langle L_{\mathrm{MM}} / \ell_{*}\right\rangle=2.3 \quad$ (see next section). Combining Eqs. (10)-(11) one sees that $c_{\tau}$ decreases with the relative magnitude of measured shear stress (as $\sigma_{\text {iso }} / u_{*, \mathrm{obs}}$ ); this is also expressed usefully through the measured ratio of streamwise fluctuation amplitude to friction velocity:

$c_{\tau} \simeq \frac{1.5 \Gamma}{c_{m}} \frac{\sigma_{\text {iso }}}{u_{*, \mathrm{obs}}}=\frac{1.5 \Gamma}{\sigma_{u, \mathrm{obs}} / \sigma_{\text {iso }}}\left(\frac{\sigma_{u, \mathrm{obs}} / u_{*, \mathrm{obs}}}{c_{m}}\right)$.

From the above and Eq. (10) one subsequently then finds

$L_{\mathrm{MM}} \simeq \frac{\sigma_{u, \mathrm{obs}}}{\mathrm{d} U / \mathrm{d} z}\left(\frac{c_{m}}{\sigma_{u, \mathrm{obs}} / u_{*, \mathrm{obs}}}\right)$.

For constant $\left(\sigma_{u, \mathrm{obs}} / u_{*, \mathrm{obs}}\right)$, Eq. (13) implies that the turbulence scale $L_{\mathrm{MM}}$ can be expressed independently of $\Gamma$, given $\sigma_{u, \text { obs }}$ and $\mathrm{d} U / \mathrm{d} z$.

Caughey et al. (1979) reported the mean profile of $\sigma_{u}^{2}(z)$ from the seminal "Kansas experiment", showing that $\left(\sigma_{u} / u_{*}\right)_{0}^{2} \approx 5-6$ in the homogeneous atmospheric surface layer (their Fig. 5). The corresponding value of $\left(\sigma_{u} / u_{*}\right)_{0}$ is approximately 2.3 ; thus, if $c_{m} \approx 2.3$ as well, then Eq. (12) reduces to

$c_{\tau} \approx \frac{1.5 \Gamma}{\sigma_{u, \mathrm{obs}} / \sigma_{\mathrm{iso}}}$.

Given the definition of $c_{\tau}$ through Eq. (7), $c_{\tau}$ is a constant; since Eq. (9) shows $\sigma_{\text {iso }}$ is independent of $\Gamma$, then $\sigma_{u \text {, obs }} \propto \Gamma$. Consistent with this argument, Eq. (13) reduces to

$L_{\mathrm{MM}} \approx \frac{\sigma_{u, \mathrm{obs}}}{\mathrm{d} U / \mathrm{d} z}$,

which is also evident inserting Eq. (14) into Eq. (10). Using Eq. (15), $L_{\mathrm{MM}}$ can simply be diagnosed from typical measurements, e.g., 10 min average cup-anemometer output at two (or more) heights. The length $L_{\mathrm{MM}}$ can also be cast in terms of variables commonly used in wind engineering, notably the turbulence intensity $I_{u}$ and shear exponent $\alpha$. Invoking $\mathrm{d} U / \mathrm{d} z=\alpha U / z$ (Kelly et al., 2014a) and defining $I_{\text {obs }} \equiv \sigma_{u, \text { obs }} / U$, then Eq. (15) becomes

$L_{\mathrm{MM}} \approx z \frac{I_{\mathrm{obs}}}{\alpha}$

\subsubsection{Modeled spectra: covariances, anisotropy, and Г}

The spectral Mann model ("MM") distorts the isotropic von Kármán spectral tensor $\left(\Phi_{i j}(\boldsymbol{k})\right.$, Eq. 4), per wavenumber via rapid-distortion theory over the wavenumber-dependent eddy lifetime $\tau_{\mathrm{M}}$, such that the component spectra become anisotropic at wavenumbers outside (lower than) the inertial range; the degree of distortion - and thus anisotropy - are consequently represented by the eddy-lifetime parameter $\Gamma$. Above we showed via mixing-length arguments that $L_{\mathrm{MM}}$ is 
independent of $\Gamma$, resulting in Eq. (15). Possible $\Gamma$ dependences can also be examined by considering the shear stress

$$
\langle u w\rangle_{\mathrm{MM}}=-u_{*, \mathrm{MM}}^{2}=2 \int_{0}^{\infty} F_{13}\left(k_{1}\right) \mathrm{d} k_{1}
$$

obtained from the modeled spectral tensor component $F_{13}\left(k_{1}\right)=\iint \Phi_{13} \mathrm{~d} k_{2} \mathrm{~d} k_{3}$, which is expected to be a function of $\Gamma$. Indeed Mann (1994, Fig. 4) shows this to be the case, with modeled stress $\langle u w\rangle_{\mathrm{MM}} / \sigma_{\text {iso }}^{2}$ varying almost linearly between 0 and -1 for $0<\Gamma<5$; then $u_{*, \mathrm{MM}}^{2} / \sigma_{\text {iso }}^{2} \approx \Gamma / 5$. Subsequently from Eq. (12) one has

$c_{\tau} \approx \frac{1.5 \sqrt{5 \Gamma}}{c_{m}} \frac{u_{*, \mathrm{MM}}}{u_{*, \mathrm{obs}}} \approx \frac{0.64 \Gamma}{u_{*, \mathrm{obs}} / \sigma_{\mathrm{iso}}}$

for $c_{m} \approx 2.3$, in analogy with Eq. (14); thus we expect $u_{*, \text { obs }} \propto \Gamma$, similar to the expected behavior of $\sigma_{u, \text { obs }} \propto \Gamma$ following Eq. (14).

In addition to the approximate expression (18), which is based on the simplified relation $u_{*, \mathrm{MM}}^{2} / \sigma_{\text {iso }}^{2} \approx \Gamma / 5$, it is possible to derive an exact relation based on the Mann-model shear stress (Eq. 17) - but this is cumbersome and analytically intractable. Though de Mare and Mann (2016) derived implicit expressions toward relating $\left\{\Gamma, \mathrm{d} U / \mathrm{d} z, L_{\mathrm{MM}}\right\}$ to the eddy lifetime and integral of the modeled stress spectrum (Eq. 17), these must be evaluated numerically or graphically. An explicit expression corresponding to $c_{m}^{-1}=$ $\ell_{*} / L_{\mathrm{MM}}$ (like Eq. 11 here) was derived by de Mare and Mann (2016), but it depends on numerically integrating the stress spectrum.

As spectra fitted to Mann-model outputs correspond to distorted anisotropic turbulence, and noting the $\Gamma$ dependence of $u_{*, \mathrm{MM}}$ discussed above, we expect $\sigma_{u, \mathrm{MM}}$ to also depend on $\Gamma$. From Fig. 4 of Mann (1994) we find $\sigma_{u, \mathrm{MM}}^{2} / \sigma_{\text {iso }}^{2} \simeq\left(1+0.14 \Gamma^{2}\right)$, which for $\Gamma \gtrsim 2$, the range corresponding to atmospheric boundary layer (ABL) observations (e.g., Sathe et al., 2013), becomes roughly $\sigma_{u, \mathrm{MM}} \approx \sigma_{\mathrm{iso}}(0.61+0.3 \Gamma)$.

\subsection{Ideal neutral surface-layer implications}

Within the atmospheric surface layer (ASL), in the homogeneous stationary limit under neutral conditions, $\mathrm{d} U / \mathrm{d} z \rightarrow u_{*} /(\kappa z)$ so that Eq. (11) reduces to $L_{\mathrm{MM}} \rightarrow c_{m} \kappa z \approx 0.92 z$. Similarly, in this "log-law regime" $\varepsilon_{\mathrm{ASL}, \mathrm{N}}=u_{*}^{3} /(\kappa z)$ so that Eq. (7) becomes $\Gamma_{\mathrm{ASL}, \mathrm{N}}=c_{\tau}(3 \alpha / 2)^{-1 / 2}\left(L_{\mathrm{MM}} / \kappa z\right)^{2 / 3}$, or equivalently $\left.L_{\mathrm{MM}}\right|_{\mathrm{ASL}, \mathrm{N}}=(3 \alpha / 2)^{3 / 4} \kappa z\left[\Gamma / c_{\tau}\right]^{3 / 2}$, which via Eq. (12) can be written

$$
\begin{aligned}
\left.L_{\mathrm{MM}}\right|_{\mathrm{ASL}, \mathrm{N}} & =\left(\frac{3 \alpha}{2}\right)^{3 / 4} \kappa z\left[\frac{c_{m} u_{*, \mathrm{obs}}}{1.5 \sigma_{\text {iso }}}\right]^{3 / 2} \\
& \simeq 1.1 \kappa z\left[\frac{c_{m}}{\sigma_{\mathrm{u}, \mathrm{obs}} / u_{*, \mathrm{obs}}} \frac{\sigma_{u, \mathrm{obs}}}{\sigma_{\text {iso }}}\right]^{3 / 2} .
\end{aligned}
$$

Thus for $c_{m}=\sigma_{\mathrm{u}, \mathrm{obs}} / u^{*}$,obs, we see that the Mann (1994) eddy-lifetime formulation (3) implies $L_{\mathrm{MM}} \rightarrow 1.1 \kappa z\left(\sigma_{u, \mathrm{obs}} / \sigma_{\text {iso }}\right)^{3 / 2}$ in the neutral ASL. Meanwhile, as noted just above, the mixing-length form (11) implies $L_{\mathrm{MM}} \rightarrow c_{m} \kappa z$; this is consistent with Eq. (19) under the condition that $\left(\sigma_{u, \text { obs }} / \sigma_{\text {iso }}\right) \simeq\left(c_{m} / 1.1\right)^{2 / 3}$ or roughly $\sigma_{u, \text { obs }} \approx 1.6 \sigma_{\text {iso }}$ for $c_{m} \simeq 2.3$.

\section{Observations and results}

Since the choice of eddy lifetime form (3) leads to a sheardependent relation (7) between the spectral-tensor model parameters, one obtains Eq. (10) for the undistorted (isotropic) length scale, with $L_{\mathrm{MM}} \propto \sigma_{\mathrm{iso}}(\mathrm{d} U / \mathrm{d} z)^{-1}$; further invoking a mixing-length argument then leads to a relation (15) for $L_{\mathrm{MM}}$ in terms of quantities that are directly measurable via standard wind-industry (one-dimensional cup) anemometers. Here we test Eq. (15) as well the assumptions leading to it, through measured wind speed, shear, and turbulent velocity component spectra. We also find a form for the distribution of $L_{\mathrm{MM}}$ over all conditions - as would be needed in practice to represent the turbulence length scales of flows experienced by wind turbines at a given site.

For the assumption testing in this section, the spectra used are measured via three-dimensional sonic anemometers on the primary meteorological mast located at the Danish National Test Centre for Large Wind Turbines (Høvsøre), $1.75 \mathrm{~km}$ from the western coast of Denmark (Mann et al., 2005; Peña et al., 2016). The anemometers give $20 \mathrm{~Hz}$ samples of all three velocity components and temperature ${ }^{8}$ at heights of $10,20,40,60,80$, and $100 \mathrm{~m}$. This allows calculation of mean speeds, directions, and vertical shear of mean speed over individual $10 \mathrm{~min}$ records; in particular we focus on heights of $z=20 \mathrm{~m}$ and $z=80 \mathrm{~m}$, as we are able to calculate shear at (across) these heights using the measurements at $10,40,60$, and $100 \mathrm{~m}$ while also using the measured wind speed components and subsequent spectra at $z=\{20,80\} \mathrm{m}$. The parameters $\left\{L_{\mathrm{MM}}, \Gamma, \epsilon\right\}$ are obtained via fits of precalculated Mann-model spectra to the measured velocity-component and stress spectra $F_{11}(f)$, $F_{22}(f), F_{33}(f)$, and $F_{13}(f)$; this is done via Taylor's hypothesis $\left(k_{1}=2 \pi f / U\right)$ along with combined least-squares fits (Mann, 1994; Chougule et al., 2017).

\footnotetext{
${ }^{8}$ The sonic anemometers actually give a temperature very close to the virtual temperature, i.e., the temperature including buoyant effects of water vapor.
} 


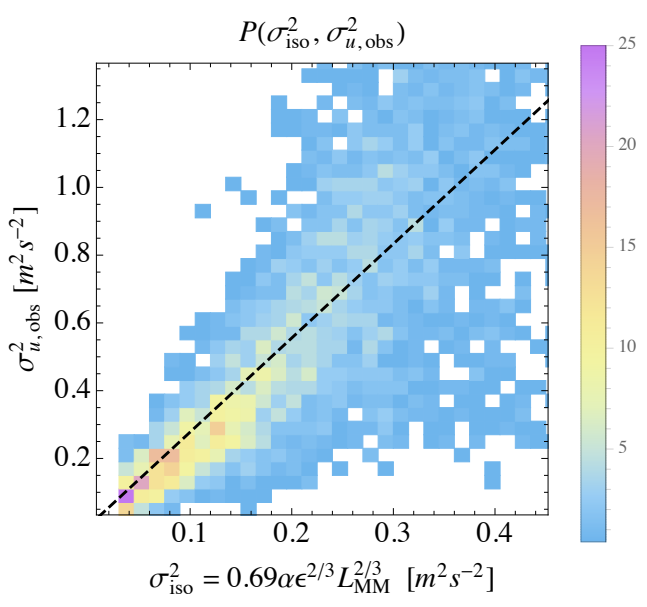

Figure 1. Joint distribution of isotropic (un-distorted) variance $\sigma_{\text {iso }}^{2}\left(\varepsilon, L_{\mathrm{MM}}\right)$ obtained from fits to measured spectra and observed streamwise variance $\sigma_{u \text {,obs }}$, from height $z=80 \mathrm{~m}$ over homogeneous land sectors at Høvsøre; dashed line corresponds to $\sigma_{u, \mathrm{obs}} / \sigma_{\text {iso }}=5 / 3$.

\subsection{Testing of assumptions and predicted constraints}

The implications of Eqs. (12)-(15) included the independence of $L_{\mathrm{MM}}$ and $c_{\tau}$ from $\Gamma$, as well as (for example) the expected dependence $\sigma_{u \text {, obs }} \propto \Gamma \sigma_{\text {iso }}$. Indeed we find that $L_{\mathrm{MM}}$ is independent of $\Gamma$, with no significant statistical correlation: $\left\langle L_{\mathrm{MM}} \Gamma\right\rangle / \sqrt{\left\langle L_{\mathrm{MM}}^{2}\right\rangle\left\langle\Gamma^{2}\right\rangle}<0.15$ for land or sea sectors at any given height. We also confirm that $\sigma_{u, \text { obs }} \propto \Gamma \sigma_{\text {iso }}$, which is demonstrated by Figs. 1-2. The first figure displays the joint probability density $P\left(\sigma_{u, \text { obs }}, \sigma_{\text {iso }}\right)$, where $\sigma_{u \text {,obs }}$ is the streamwise turbulent variance measured in 10 min intervals, and $\sigma_{\text {iso }}$ is calculated using Eq. (9) with $L_{\mathrm{MM}}$ and $\epsilon$ from spectral fits corresponding to the same intervals. One can see from Fig. 1 that $\sigma_{u \text {,obs }}$ generally follows $\sigma_{\text {iso }}$, and we find $\sigma_{u, \text { obs }} / \sigma_{\text {iso }} \approx 5 / 3$. Such evidence corresponds closely to the predicted constraint following Eq. (19) that $\sigma_{u \text {,obs }} / \sigma_{\text {iso }}$ should have a value of roughly 1.6 in the neutral surface layer; this is reasonable in the mean, since conditions on average are essentially neutral due to the shape of the stability distribution at Høvsøre (Kelly and Gryning, 2010). Figure 2 further shows that $\sigma_{u \text {,obs }} / \sigma_{\text {iso }} \propto \Gamma$, consistent with $c_{\tau}$ being a constant independent of $\Gamma$ following Eq. (14). The slope of the line in Fig. 2 also corresponds to the approximate Mann-model behavior $\sigma_{u, \mathrm{MM}} \approx \sigma_{\text {iso }}(0.61+0.3 \Gamma)$ for $\Gamma \gtrsim 2$, outlined at the end of Sect. 2.2.2 above.

Considering wind speeds in the typical turbine operating range of 4-25 m s${ }^{-1}$, the Høvsøre data also confirm that $\left\langle\sigma_{u} / u_{*}\right\rangle_{\text {obs }} \approx 2.3$, consistent with the findings of Caughey et al. (1979). Further, the data also show that $\left\langle c_{m}\right\rangle \approx 2.3$ so that Eq. (13) reduces approximately to Eq. (15). It is also found that the same approximate trends are seen when considering only $U>7 \mathrm{~m} \mathrm{~s}^{-1}$ (not shown), but with slightly less scatter (narrower joint distributions) away from the predicted

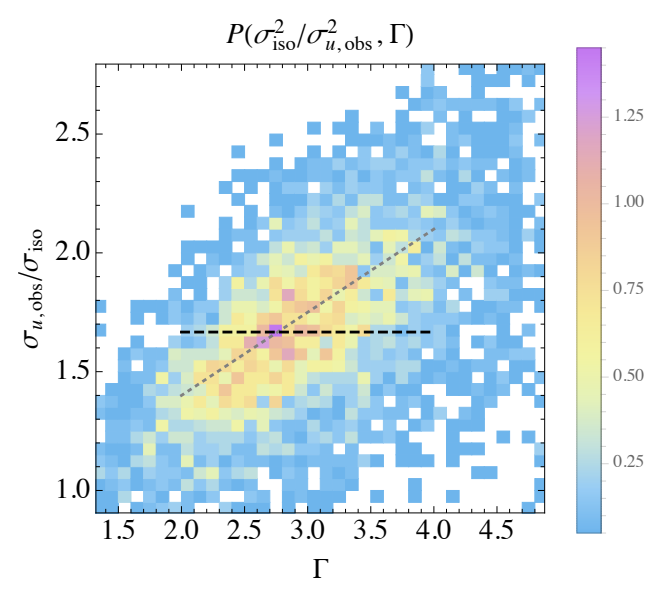

Figure 2. Ratio of observed streamwise to isotropic fluctuation magnitude versus $\Gamma$ obtained from spectral fits, plotted as joint probability density function $P\left(\Gamma, \sigma_{u, \text { obs }} / \sigma_{\text {iso }}\right)$. Dashed (horizontal) line shows $\sigma_{u, \mathrm{obs}} / \sigma_{\text {iso }}=\sqrt{5 / 3}$ corresponding to slope of dashed line in Fig. 1; dotted line shows the mean linear $\Gamma$ dependence of $\sigma_{u, \mathrm{obs}} / \sigma_{\mathrm{iso}}$.

$\sigma_{u, \text { obs }} / \sigma_{\text {iso }}$ behaviors shown in Figs. 1-2 (dashed/dotted lines) and discussed above.

The data also show that $\sigma_{u} / u_{*}$ is not correlated with $L_{\mathrm{MM}}$, whether we include all speeds or limit the wind speed range to $7-25$ or $4-25 \mathrm{~m} \mathrm{~s}^{-1}$. Thus this ratio can be treated as a constant in Eq. (13) for a given height (or throughout the surface layer), using Eq. (13) over a range of wind speeds.

\subsection{Turbulence length-scale distributions $P\left(L_{\mathrm{MM}}\right)$}

The efficacy of using Eq. (15) to estimate the spectral length scale $L_{\mathrm{MM}}$ can be seen by considering Fig. 3. The figure displays the joint distribution of turbulence length scale at a height of $z=80 \mathrm{~m}$, i.e., $P\left(L_{\mathrm{MM}, \mathrm{obs}}, \sigma_{u, \mathrm{obs}}|\mathrm{d} U / \mathrm{d} z|^{-1}\right)$; this is obtained through Eq. (15) from $10 \mathrm{~min}$ measurements and via fitting observed spectra. Figure 3 is usefully interpreted as the probability-weighted performance of Eq. (15) for predicting $L_{\mathrm{MM}}$ (from $\sigma_{u \text {, obs }}$ measured at $z=80 \mathrm{~m}$ and the shear $\mathrm{d} U / \mathrm{d} z$ observed over $z=60-100 \mathrm{~m})$ versus the $L_{\mathrm{MM}}$ obtained from fits of the spectral-tensor model to corresponding 10 min spectra. One sees a 1:1 relationship, particularly for the most commonly found values of the length scale; these $L_{\text {MM }}$ values range $\sim 15-50 \mathrm{~m}^{9}$. Compared to the scales calculated from observed spectra, there is some mis-prediction of $L_{\mathrm{MM}}$ calculated by Eq. (15), but it is relatively rare; this

\footnotetext{
${ }^{9}$ The spectral fits were done using spectral-tensor model output over the parameter ranges of $5<L_{\mathrm{MM}}<500 \mathrm{~m}$ and $0 \leq \Gamma \leq 5$. Some spectra were poorly fitted; since these occurred when $\Gamma=5$, cases with $\Gamma>4.95$ were excluded from the analysis here. As justification, I note that only a small fraction of the cases $(<10 \%)$ had such $\Gamma$, and we only consider well-fit spectra for reliable comparison of parameters.
} 


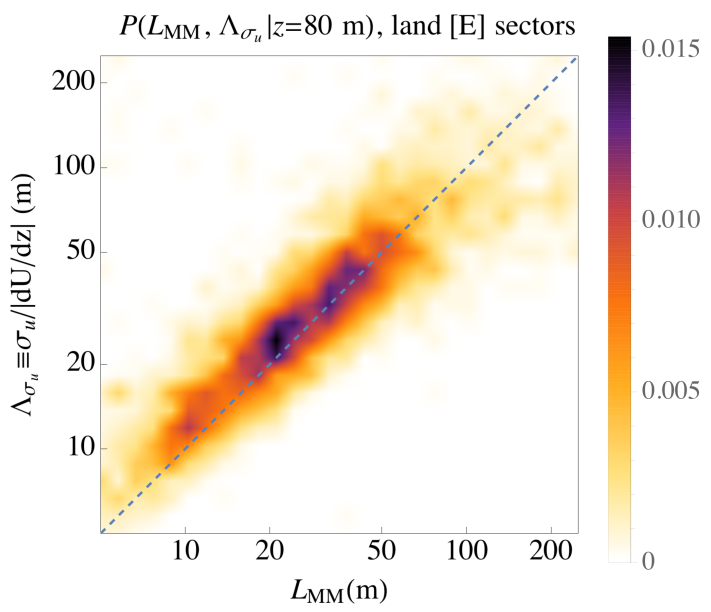

Figure 3. Joint probability density function of predicted and diagnosed (observed) turbulent length scale, from measurements at Høvsøre over the homogeneous eastern land sectors. $x$ axis: Mannmodel scale $L_{\mathrm{MM}}$ from spectral fits; $y$ axis: $L_{\mathrm{MM}}$ estimated from direct measurements of $\mathrm{d} U / \mathrm{d} z$ and $\sigma_{u}$, via Eq. (15).

is shown by the low probabilities in Fig. 3 away from the well-predicted, most commonly occurring $L_{\mathrm{MM}}$.

To demonstrate the statistical character of Eq. (15), as well as its potential for probabilistic use (e.g., as input to probabilistic load calculations), Fig. 4 shows the probability density $P\left(L_{\mathrm{MM}}\right)$. As in Fig. $3, L_{\mathrm{MM}}$ is again calculated from fits to 10 min spectra and also estimated by $\sigma_{u, \text { obs }} /(\mathrm{d} U / \mathrm{d} z)$, i.e., Eq. (15). Additionally Fig. 4 displays $P\left(L_{\mathrm{MM}}\right)$ for $L_{\mathrm{MM}}$ calculated through Eq. (11), i.e., $c_{m} u_{*} /(\mathrm{d} U / \mathrm{d} z)$; this is done both using the value of $c_{m}=1.7$ reported by Peña et al. (2010) as well as using the approximate mean of 2.3 found to be consistent with measurements and theory in Sects. 3.1 and 2.2 above. From Fig. 4 one sees that, for values of turbulent peak scale greater than the mode $(\sim 20 \mathrm{~m})$ up to roughly $150 \mathrm{~m}$, there is a match between the distribution of the diagnosed $L_{\mathrm{MM}}$ and distributions of length scale estimated from the forms (15) based on $\sigma_{u, \text { obs }}$ and (11) based on $u_{*}$ with $c_{m}=2.3$; these are roughly equivalent for this case over relatively simple homogeneous terrain. It is found that the Peña et al. (2010) value of $c_{m}=1.7$ leads to overprediction of $L_{\mathrm{MM}}$ by a factor of 2 or more at scales smaller than $10 \mathrm{~m}$ and underprediction by $50 \%$ or more at scales larger than $50 \mathrm{~m}$. The $u_{*}$-based form (11) using $c_{m}=2.3$ matches the spectrally fit diagnosed distribution $P\left(L_{\mathrm{MM}}\right)$ slightly better than the $\sigma_{u}$-based form (15), with predicted peak (mode) values of $L_{\mathrm{MM}}$ being about 3-4 m smaller than the diagnosed peak $L_{\mathrm{MM}}$.

For the homogeneous land case in Fig. 4 the probability density function of $2.3 u_{*, \text { obs }} /(\mathrm{d} U / \mathrm{d} z)$ matches $P\left(L_{\mathrm{MM}}\right)$ observed from the spectral fits to within $10 \%$, over the range $10 \mathrm{~m} \lesssim L_{\mathrm{MM}} \lesssim 75 \mathrm{~m}$, and the probability density function of $\sigma_{u, \text { obs }} /(\mathrm{d} U / \mathrm{d} z)$ also matches within $10 \%$ over the range $15 \mathrm{~m} \lesssim L_{\mathrm{MM}} \lesssim 50 \mathrm{~m}$. This is consistent with the darkly col-

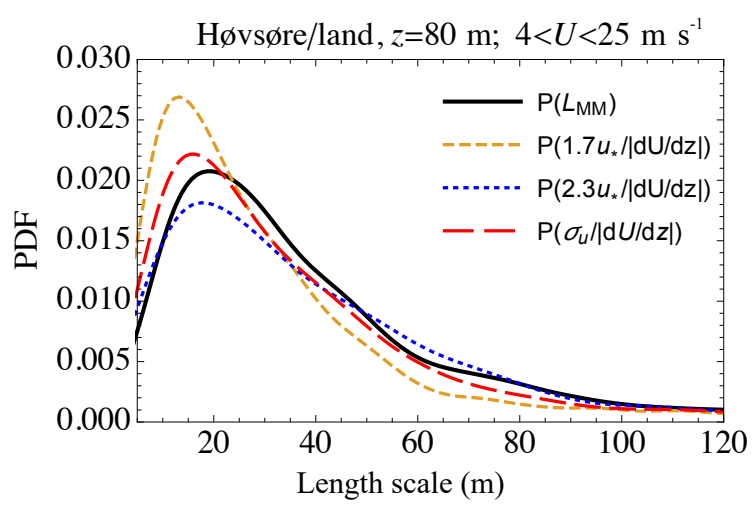

Figure 4. Probability density function of turbulent length scale from observations at Høvsøre from the homogeneous eastern land sectors. Black: Mann-model scale from fits to spectra; dotted/blue: "mixing-length" formulation $\left(\ell_{m} \propto u_{*} /|\mathrm{d} U / \mathrm{d} z|\right)$ with revised constant; dashed/gold: Peña et al. (2010) form for $\ell_{m}$; red/long-dashed: $\sigma_{u} /|\mathrm{d} U / \mathrm{d} z|$ form (15).

ored $1: 1$ patch evident in Fig. 3 and also shows that Eq. (15) (and also Eq. 11 with $c_{m}=2.3$ ) is sufficient for probabilistic wind load simulations, for two reasons. First, the wellmatched range of scales corresponds to the most commonly found $L_{\mathrm{MM}}$. Secondly, although scales smaller than $\sim 15 \mathrm{~m}$ are not rare (with an occurrence of roughly 1 in 6), they will have a diminishing effect on turbine loads. More specifically, $L_{\mathrm{MM}}$ is more than $70 \%$ likely to fall in the $15-75 \mathrm{~m}$ range, i.e., $P\left(15 \mathrm{~m}<L_{\mathrm{MM}}<75 \mathrm{~m}\right)>0.7$, and $L_{\mathrm{MM}}$ has more than $86 \%$ likelihood of occurrence between 0 and $75 \mathrm{~m}$, for this homogeneous land case at $z=80 \mathrm{~m}$. The relatively common shorter scales correspond to weaker turbulent fluctuations (thus loads), because on average $\sigma_{u, \mathrm{obs}} \propto L_{\mathrm{MM}}^{2 / 3}$, as implied by Fig. 1 and Eqs. (9)-(15). Further, turbine loads are less influenced by fluctuations characterized by spatial scales significantly smaller than the blade lengths; thus the error in predicted probability for these shorter scales, and the slight underprediction of the most common $L_{\mathrm{MM}}$, should not significantly influence probabilistic load calculations relying on site-specific $L_{\mathrm{MM}}$ obtained via measurements and Eq. (15).

While Eq. (15) is useful to estimate $L_{\mathrm{MM}}$ and $P\left(L_{\mathrm{MM}}\right)$ as shown above, one expects Eq. (13) to perform better, as it does not rely on the approximation $c_{m}=\sigma_{u} / u_{*}$. Indeed $\left\langle c_{m} u_{*} / \sigma_{u}\right\rangle$ is actually 1.11 (or 1.13 if considering winds only down to $7 \mathrm{~m} \mathrm{~s}^{-1}$ ) due to $\sigma_{u} / u_{*}$ being slightly smaller and $c_{m}$ slightly larger than 2.3; using these values in Eq. (13) gives estimates of $L_{\mathrm{MM}}$ closer to the spectrally diagnosed $L_{\mathrm{MM}}$, and within $10 \%$ of $P\left(L_{\mathrm{MM}}\right)$ over a range of $L_{\mathrm{MM}}$ from below $10 \mathrm{~m}$ to beyond $100 \mathrm{~m}$. It should also be noted that ignoring speeds below $7 \mathrm{~m} \mathrm{~s}^{-1}$ can lead to slightly smaller $L_{\mathrm{MM}}$, since these low wind speeds are more influenced by unstable conditions. Indeed for $L_{\mathrm{MM}} \gtrsim 50 \mathrm{~m}$, including the lower wind speeds causes both diagnosed and predicted $L_{\mathrm{MM}}$ to increase 
roughly $10 \%$; this is consistent with larger turbulent eddies being created under unstable conditions.

\subsubsection{Estimating $P\left(L_{\mathrm{MM}}\right)$ in coastal/offshore conditions}

To demonstrate the (probabilistic) use of Eq. (13) or Eq. (15) for $L_{\mathrm{MM}}$ in somewhat different conditions, we now consider flow from offshore, using data from the same mast and height as above (Høvsøre, $z=80 \mathrm{~m}$ ) but for wind directions between 240 and $300^{\circ}$. The mast is roughly $1.75 \mathrm{~km}$ east of the coastline and subsequently $1.65 \mathrm{~km}$ east of a $16-17 \mathrm{~m}$ high sand dune that lies $100 \mathrm{~m}$ inland, where both are locally oriented in the $\mathrm{N}-\mathrm{S}$ direction (i.e., for the range of wind directions considered). The dune causes enhanced/accelerated transition of the flow from an offshore (water roughness) to an over-land flow regime (Berg et al., 2015); this results in winds which reflect on-shore and coastal conditions at low heights (below $\sim 40-80 \mathrm{~m}$ depending on stability) and offshore conditions at higher $z$.

Figure 5 displays the distribution $P\left(L_{\mathrm{MM}}\right)$ of spectralpeak (Mann model) length scales for coastal/offshore winds (from west $\pm 30^{\circ}$ ), again using Eq. (15) to estimate $L_{\mathrm{MM}}$ along with $L_{\mathrm{MM}}$ diagnosed through spectral fits. For comparison the corresponding $P\left(L_{\mathrm{MM}}\right)$ for easterly winds from Fig. 4 is also included. Just as for the homogeneous land case shown in Fig. 4, one sees in Fig. 5 that, for inhomogeneous coastal conditions, again Eq. (15) gives $P\left(L_{\mathrm{MM}}\right)$ basically matching the spectrally fit observations for scales beyond $\sim 15 \mathrm{~m}$; in this coastal regime the range of well-predicted $L_{\mathrm{MM}}$ extends further, to $\sim 150 \mathrm{~m}$. While one sees that the distribution of $L_{\mathrm{MM}}$ is a bit different for the (western) inhomogeneous coastal case than for the (eastern) homogeneous land case, the simple expression (15) functions similarly for both flow regimes, with the arguments presented in Sect. 3.2 again applying here.

The $u_{*}$-based Eq. (11) also behaves similarly (not shown) as in the homogeneous land case of Fig. 4, i.e., with gross overpredictions at small scales and underpredictions at large scales. One difference between the coastal and land cases is that, for small $L_{\mathrm{MM}}$, Eq. (15) overestimates the distribution $P\left(L_{\mathrm{MM}}\right)$ a bit more for the coastal regime than for the homogeneous land regime $\left(L_{\mathrm{MM}}<20 \mathrm{~m}\right)$; as explained above for the land case, an overprediction at the smallest scales is not expected to significantly impact load calculations, due to the relatively small length scales involved.

\subsubsection{Estimation of $P\left(L_{\mathrm{MM}}\right)$ in more complex conditions}

To further show the behavior of $L_{\mathrm{MM}}$ and the utility of Eq. (15) at a site with more complex conditions, we examine data from the inhomogeneous forested Danish National Test Centre for Large Wind Turbines site near Østerild in Denmark (e.g., Hansen et al., 2014, for details). Here sonic anemometer data are available at heights of 10 and $44 \mathrm{~m}$, with concurrent data from three lidars

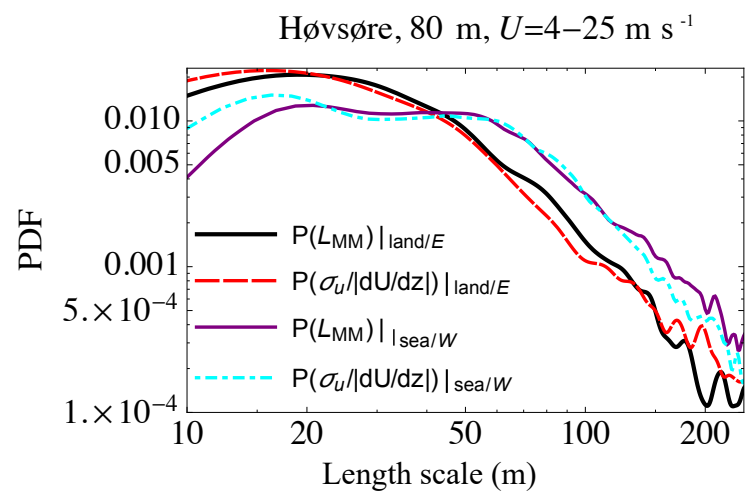

Figure 5. Probability density of turbulence length scale $L_{\mathrm{MM}}$ from observations at Høvsøre over both the homogeneous land (eastern) sectors and inhomogeneous coastal (western) sectors. Black: $L_{\mathrm{MM}}$ from fits to spectra over land; red/long-dashed: new simplified form (15) over land; purple: $L_{\mathrm{MM}}$ from fits to spectra from offshore; cyan/dot-dashed: new simplified form (15) from offshore.

at $z=\{45,80,140,200,300\} \mathrm{m}$. In this study we consider data from the site's "western lidar" 10 , to measure winds that flow over the forest more than $70 \%$ of the time, where the canopy height is 10-20 m (Hansen et al., 2014; Sogachev et al., 2017). The analysis here uses one year (May 2010-May 2011) of wind speeds $U \geq 5 \mathrm{~m} \mathrm{~s}^{-1}$ from the lidar at 45 and $80 \mathrm{~m}$ heights along with the "fast" $(20 \mathrm{~Hz})$ data from the sonic anemometer at $44 \mathrm{~m}$. The shear $\mathrm{d} U / \mathrm{d} z$ is measured across $45-80 \mathrm{~m}$; the spectra and subsequent turbulence/Mann-model parameters $\left\{L_{\mathrm{MM}}, \Gamma, \varepsilon\right\}$, as well as and measured quantities $\left\{\sigma_{u, \mathrm{obs}}, u_{* \text {,obs }}\right\}$, are obtained from the sonic anemometer. The measurements are significantly higher than twice the forest canopy height, and thus above the roughness sublayer (Garratt, 1980; Raupach et al., 1980) and amenable to similarity and mixing-length theory (e.g., Sogachev and Kelly, 2016) as well as Mann-model use (Chougule et al., 2015).

Just as Fig. 4 showed for flow over homogeneous land at Høvsøre in Sect. 3, here Fig. 6 displays the probability density of turbulence (Mann-model) length scale $L_{\mathrm{MM}}$ observed via spectral fits at $z=44 \mathrm{~m}$ for $\emptyset$ sterild, along with predictions based on both Eq. (11) via $u_{* \text {,obs }}$ and Eq. (15) via $\sigma_{u, \text { obs. }}$

As in the cases above (homogeneous land and inhomogeneous coastal), the new form (15) predicts the distribution rather well, particularly for scales $\sim 10-100 \mathrm{~m}-$ despite the shape of $P\left(L_{\mathrm{MM}}\right)$ being different due to the trees. For the forest case of Fig. 6 the $\sigma_{u}$-based form captures both the peak (most likely $L_{\mathrm{MM}}$ ) and magnitude of $P\left(L_{\mathrm{MM}}\right)$, while the $u_{*}$-based form grossly underpredicts $L_{\mathrm{MM}}$, more so than for the previous cases. The latter is likely due to $u_{*, \text { obs }}$ be-

\footnotetext{
${ }^{10}$ The "western lidar" at Østerild is located $\sim 1 \mathrm{~km}$ west of the northernmost turbines but less than $100 \mathrm{~m}$ east of a forest patch and $5-20 \mathrm{~km}$ from the North Sea coastline in the prevailing (W-NW) wind directions (Hansen et al., 2014).
} 


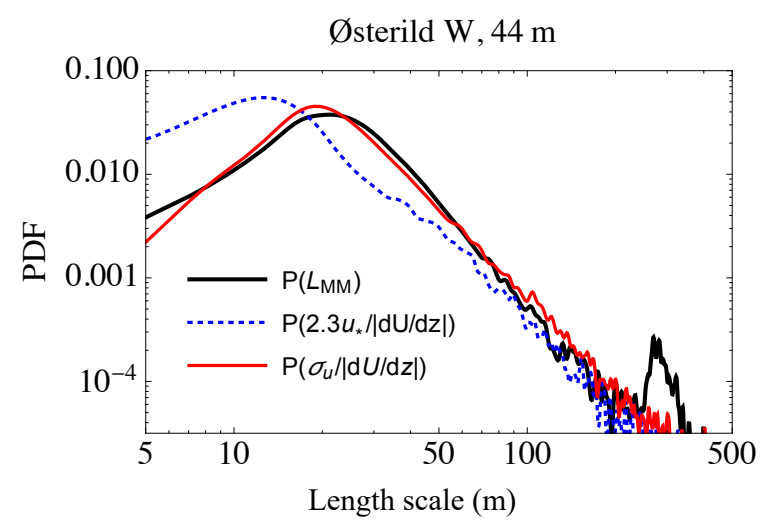

Figure 6. Probability density function of turbulent length scale from observations at Østerild from the western mast/lidar. Black: Mann-model scale from fits to spectra; dotted-blue: "mixinglength" formulation $\left(\ell_{m} \propto u_{*} /|\mathrm{d} U / \mathrm{d} z|\right)$ with revised constant; red: new form (15), $\sigma_{u} /|\mathrm{d} U / \mathrm{d} z|$.

ing predominantly affected by the canopy (via larger effective roughness) more so than $\sigma_{u \text {,obs }}$, which tends to be more characteristic of the entire ABL (Wyngaard, 2010). There is, however, a curious minor peak (with a probability $\sim 1 \%$ as large as the main peak) around scales of $\sim 300 \pm 50 \mathrm{~m}$ in the length-scale distribution obtained from spectral fits shown in Fig. 6; this is captured by neither the $u_{*}$-based form (11) nor $\sigma_{u}$-based formulations (13) and (15). Although this peak falls spectrally at small wavenumbers that are more difficult to capture when spectrally fitting the Mann model, it actually corresponds to the distance to the next upwind edge of the forest (orchard segment) in the predominant wind directions.

\section{Discussion}

Towards concluding, we first revisit the motivation for (and thus context of) this work: (1) to "close" the Mann (1994) eddy-lifetime $\left(\tau_{\mathrm{M}}\right)$ formulation as implemented in rapiddistortion theory - allowing relation between Mann-model parameters $\left(L_{\mathrm{MM}}, \varepsilon, \Gamma\right)$ and the shear $(\mathrm{d} U / \mathrm{d} z)$ taken to distort the modeled turbulence; (2) to connect the parameters of the Mann (1994) spectral turbulence and eddy-lifetime models with atmospheric statistics, both in theory and in practice; (3) to provide a formulation for the turbulence length scale $L_{\mathrm{MM}}$ in terms of quantities commonly measured in wind energy; and (4) to demonstrate that the "measurable" form developed for $L_{\mathrm{MM}}$ is robust and amenable to use in (probabilistic) wind turbine load calculations. These four motivating goals have basically been realized, as shown in the previous sections, and this work has a number of implications.

\section{Implications and application}

A previously suggested form (11) for $L_{\mathrm{MM}}$, based on friction velocity $u_{*}$ and $(10 \mathrm{~min})$ mean wind shear $\mathrm{d} U / \mathrm{d} z$ (Peña et al., 2010), was confirmed here to be sensitive to its proportionality constant $c_{m}$. But this constant can vary from site to site (and possibly with height), and the published value of $c_{m}=1.7$ (Peña et al., 2010) leads to significant error in prediction of $L_{\mathrm{MM}}$ for the different conditions (land and sea directions) at Høvsøre and at the forested site of Østerild. Finding $c_{m}$ from sonic anemometer observations via $L_{\mathrm{MM}}$ from fits to spectra and friction velocity measurements, Eq. (11) may perform slightly better over uniform flat terrain compared to the $\sigma_{u}$-based form (15) - but this can be considered a site-dependent fit in itself, as was the case when using a diagnosed value of $c_{m}=2.3$ for the homogeneous flat land sectors at Høvsøre. However, obtaining $c_{m}$ is generally not possible in industrial practice; where it can be obtained, it relies on $L_{\mathrm{MM}}$ - which is the quantity desired - thus negating the purpose of Eq. (11). While $u_{*}$ can also in principle be estimated from wind speeds taken at multiple heights by cup anemometers, this too is difficult in practice: one must account for stability, not to mention the need for measurements at multiple heights in the surface layer (or worse, the limited validity of similarity theory above the ASL). Furthermore, it is expected that $c_{m}$ is a function of the (local) surface roughness, as demonstrated by the different results found over the forested Østerild site. Thus the form (15) is preferable, since it requires only the commonly measured quantities $\sigma_{u}$ and $\mathrm{d} U / \mathrm{d} z$. This simple form also gave good estimates of $P\left(L_{\mathrm{MM}}\right)$ in the forested case - without the need for tuning, whereas the $u_{*}$-based form (11) requires a recalculation of its coefficient $c_{m}$ for such cases.

Since Eq. (13) gave yet better performance than both its simplified form (15) and the $u_{*}$-based relation (11), one might suggest its use. But Eq. (13) requires $c_{m} /\left(\sigma_{u} / u_{*}\right)$, where $c_{m}$ is difficult to obtain, as discussed in the previous paragraph. However, although $c_{m}$ might vary from site to site (or perhaps with height), it was found that the ratio $c_{m} /\left(\sigma_{u} / u_{*}\right)$ did not vary appreciably - consistent with the good performance of the simplified form (15), which assumes $c_{m} \approx \sigma_{u} / u_{*}$, across sites and regimes.

One interesting implication of the testing of assumptions then follows from the finding that $\left\langle\sigma_{u} / u_{*}\right\rangle_{\text {obs }} \approx 2.3$, consistent in the surface layer with Caughey et al. (1979). Examining the joint behavior of $\sigma_{u} / u_{*}$ and the stability parameter (inverse Obukhov length) $L^{-1}$, the sonic anemometer data available at multiple heights in this study show no correlation between these two quantities. The dimensionless profiles $\sigma_{u}^{2}(z) / u_{* 0}^{2}$ and $u_{*}^{2}(z) / u_{* 0}^{2}$ shown by Caughey et al. (1979) also imply

$\frac{\sigma_{u}^{2}(z)}{u_{*}^{2}(z)} \approx(2.3)^{2}$,

with the ratio converging to a constant above the surface layer $(z \gtrsim 0.1 h$, where the atmospheric boundary-layer depth $h$ typically ranges from $\sim 200 \mathrm{~m}$ in stable conditions to $1 \mathrm{~km}$ or more in convective conditions). The flat-terrain Høvsøre 
data in fact show the mean value $\left\langle\sigma_{u} / u_{*}\right\rangle_{\text {obs }}$ to be independent of $z$. If one knew how $c_{m}$ varied with height (and stability), then one could also use Eqs. (20) and (13) from measurements at one height range, to estimate $L_{\mathrm{MM}}$ at higher $z$ (for a given stability range). Over flat terrain, on average the peak spectral scale for streamwise fluctuations $\left(\lambda_{u}\right)$ grows with $z$ (Caughey et al., 1979; Peltier et al., 1996) ${ }^{11}$. Therefore, if we take $L_{\mathrm{MM}} \propto \lambda_{u}$, then with Eq. (20) one expects the ratio $c_{m} /\left(\sigma_{u} / u_{*}\right)$ to increase with $z$ as well. Thus from (13) the Mann-model length scale $L_{\mathrm{MM}}$ will increase with height relative to the mixing length $\ell_{*} \equiv u_{*} /(\mathrm{d} U / \mathrm{d} z)$, so at higher $z$ one would expect the general form (13) to be yet more accurate than its approximate form (15); however, this is not likely for wind turbine rotor heights, except in very stable conditions (Kelly et al., 2014b; Liu and Liang, 2010). Unfortunately the sonic-anemometer measurements available for this study did not include heights well beyond the surface layer, so such variation was difficult to detect.

It is also notable that Fig. 3 appears to imply the relative error (e.g., in \%) in estimating $L_{\mathrm{MM}}$ with Eq. (15) grows for less common values of $L_{\mathrm{MM}}$, particularly very large scales (and also at very small scales if including $U<7 \mathrm{~m} \mathrm{~s}^{-1}$ ). Thus Eq. (15) is recommended first for estimation of $P\left(L_{\mathrm{MM}}\right)$. However, the error at large scales is in part dependent on the limited (10 min) sample lengths and the fitting routine, as there are very few points to fit at the lowest frequencies. Use of 30 min samples can reduce such scatter, and modification of the fitting algorithms may also improve estimations of the larger scales.

Ongoing work includes wind-speed-dependent prediction of $L_{\mathrm{MM}}$, particularly the conditional statistics $P\left(L_{\mathrm{MM}} \mid U\right)$. Further concurrent work also entails systematic accounting for the rotor size (shear distance) relative to height (i.e., $\Delta z / z$ ) within the distribution of length scales; following Kelly and Gryning (2010) and Kelly et al. (2014a) a semiempirical derivation of $P\left(L_{\mathrm{MM}}\right)$ including $\Delta z / z$ has been obtained but demands more data for validation and publication. Understanding of the latter facilitates "vertical extrapolation" of $L_{\mathrm{MM}}$ and measured turbulence and shear statistics, as well as accounting for the effect of rotor size or shear measurement span.

\section{Conclusions}

- The eddy lifetime of Mann (1994), which is part of commonly used turbulence modeling for wind turbine design load cases (e.g., IEC 61400-1, Edition 3, 2005),

\footnotetext{
${ }^{11}$ The peak length scale also grows with boundary-layer depth $h$ in convective conditions and thus with increasingly negative inverse Obukhov length $L^{-1}$ (e.g., Peltier et al., 1996). But over all stability conditions, which are dominated by neutral conditions (Kelly and Gryning, 2010), and over an expected distribution of $h$ at a given site, the basic growth of $\lambda_{u}$ with $z$ is consistent with Peltier et al. (1996) reporting $\lambda_{u} \propto z$ for neutral conditions.
}

leads to a relation for turbulence (spectral-peak) length scale $L_{\mathrm{MM}}$ of

$$
L_{\mathrm{MM}} \simeq \frac{c_{m}}{\left(\sigma_{\mathrm{u}, \mathrm{obs}} / u_{*, \mathrm{obs}}\right)} \frac{\sigma_{u}}{\mathrm{~d} U / \mathrm{d} z},
$$

where $c_{m}$ and $\sigma_{u, \mathrm{obs}} / u_{*, \mathrm{obs}}$ are essentially constants for a given height $z$, and $c_{m} /\left(\sigma_{u \text {,obs }} / u_{*, \text { obs }}\right)$ is found to fall between 1 and 1.11 for the three flow regimes analyzed.

- Theory and measurements support the assumption that $c_{m} /\left(\sigma_{\mathrm{u}, \mathrm{obs}} / u *\right.$ obs $) \approx 1$, roughly constant for different atmospheric flow regimes; the turbulence length scale can consequently be approximated by

$$
L_{\mathrm{MM}} \simeq \frac{\sigma_{u}}{\mathrm{~d} U / \mathrm{d} z} .
$$

Thus typical 10 min mean cup anemometer measurements can be used to estimate $L_{\mathrm{MM}}$.

- $L_{\mathrm{MM}}$ is affected by atmospheric stability; this effect is contained within $\sigma_{u}$ and $\mathrm{d} U / \mathrm{d} z$.

- In terms of the classic mixing-length form $u_{*} /|\mathrm{d} U / \mathrm{d} z|$, the turbulence length scale $L_{\mathrm{MM}}$ in the spectral-tensor model is observed to be larger (by ca. 30-40\%) than previously reported by Peña et al. (2010).

Data availability. The data are within an SQL database at DTU and are not publicly available.

Competing interests. The author declares that he has no conflict of interest.

Acknowledgements. The author thanks the reviewers for their time and effort towards constructive criticism of the present article, and thanks are owed to Nikolay Dimitrov for discussions around probabilistic loads. This work was partly supported by the DTU Wind Energy internally funded cross-sectional project "Wind to Loads".

Edited by: Horia Hangan

Reviewed by: two anonymous referees

\section{References}

Abramowitz, M. and Stegun, I. A.: Handbook of Mathematical Functions with Formulas, Graphs, and Mathematical Tables; 9th printing, Dover, New York, 1972.

Andre, J. C. and Lesieur, M.: Influence of helicity on the evolution of isotropic turbulence at high Reynolds number, J. Fluid Mech., 81, 187-207, https://doi.org/10.1017/S0022112077001979, 1977. 
Berg, J., Vasiljevic, N., Kelly, M. C., Lea, G., and Courtney, M.: Addressing Spatial Variability of Surface-Layer Wind with LongRange WindScanners, J. Atmos. Ocean. Tech., 32, 518-527, https://doi.org/10.1175/JTECH-D-14-00123.1, 2015.

Caughey, S., Wyngaard, J. C., and Kaimal, J.: Turbulence in the evolving stable boundary-layer, J. Atmos. Sci., 36, 1041-1052, 1979.

Chougule, A., Mann, J., Segalini, A., and Dellwik, E.: Spectral tensor parameters for wind turbine load modeling from forested and agricultural landscapes, Wind Energy, 18, 469-481, 2015.

Chougule, A. S., Mann, J., Kelly, M., and Larsen, G. C.: Modeling Atmospheric Turbulence via Rapid Distortion Theory: Spectral Tensor of Velocity and Buoyancy, J. Atmos. Sci., 74, 949-974, https://doi.org/10.1175/JAS-D-16-0215.1, 2017.

Comte-Bellot, G. and Corrsin, S.: Simple Eulerian time correlation of full- and narrow-band velocity signals in grid-generated, "isotropic" turbulence, J. Fluid Mech., 48, 273-337, 1971.

de Mare, M. T. and Mann, J.: On the Space-Time Structure of Sheared Turbulence, Bound.-Lay. Meteorol., 160, 453-474, https://doi.org/10.1007/s10546-016-0143-z, 2016.

Dimitrov, N. K., Natarajan, A., and Kelly, M.: Model of Wind Shear Conditional on Turbulence and its impact on Wind Turbine Loads, Wind Energy, 18, 1917-1931, https://doi.org/10.1002/we.1797, 2015.

Dimitrov, N. K., Natarajan, A., and Mann, J.: Effects of normal and extreme turbulence spectral parameters on wind turbine loads, Renew. Energ., 101, 1180-1193, 2017.

Garratt, J. R.: Surface Influence Upon Vertical Profiles in the Atmospheric Near-Surface Layer, Q. J. Roy. Meteor. Soc., 106, 803819, https://doi.org/10.1002/qj.49710645011, 1980.

Hansen, B. O., Courtney, M., and Mortensen, N. G.: Wind Resource Assessment - Østerild National Test Centre for Large Wind Turbines, Tech. Rep. DTU Wind Energy E-0052(EN), Ris $\varnothing$ Lab/Campus, Danish Tech. Univ. (DTU), Roskilde, Denmark, 2014.

IEC 61400-1, Edition 3: Wind turbine generator systems - Part 1: Safety requirements, International Electrotechnical Comission, Geneva, Switzerland, 2005.

Kaimal, J. C., Wyngaard, J. C., Izumi, Y., and Coté, O. R.: Spectral characteristics of surface-layer turbulence, Q. J. Roy. Meteor. Soc., 98, 563-589, 1972.

Kelly, M. and Gryning, S.-E.: Long-Term Mean Wind Profiles Based on Similarity Theory, Bound.-Lay. Meteorol., 136, 377390, 2010.

Kelly, M., Larsen, G., Dimitrov, N. K., and Natarajan, A.: Probabilistic Meteorological Characterization for Turbine Loads, J. Phys. Conf. Ser., 524, 012076, https://doi.org/10.1088/17426596/524/1/012076, 2014a.

Kelly, M., Troen, I., and Jørgensen, H. E.: Weibull- $k$ revisited: "tall” profiles and height variation of wind statistics, Bound.-Lay. Meteorol., 152, 107-124, 2014b.

Lesieur, M.: Turbulence in fluids: stochastic and numerical modelling, Springer, Dordrecht, NL, https://doi.org/10.1007/978-94009-0533-7, 1990.
Liu, S. and Liang, X.-Z.: Observed Diurnal Cycle Climatology of Planetary Boundary Layer Height, J. Climate, 23, 5790-5809, 2010.

Mann, J.: The spatial structure of neutral atmospheric surface-layer turbulence, J. Fluid Mech., 273, 141-168, 1994.

Mann, J.: The spectral velocity tensor in moderately complex terrain, J. Wind Eng., 88, 153-169, 2000.

Mann, J., Ott, S., Jørgensen, B. H., and Frank, H. P.: WAsP Engineering 2000, Tech. Rep. Risø-R-1356(EN), Risø National Laboratory, Roskilde, Denmark, 2002.

Mann, J., Astrup, P., Jensen, N., Landberg, L., and Jørgensen, H.: The meteorology of "the very large wind turbines", in: Proc. of the 2004 European Wind Energy Conference, European Wind Energy Association (EWEA), London, 2005.

Peltier, L. J., Wyngaard, J. C., Khanna, S., and Brasseur, J. G.: Spectra in the Unstable Surface Layer, J. Atmos. Sci., 53, 4961, 1996.

Peña, A., Gryning, S.-E., and Mann, J.: On the length-scale of the wind profile, Q. J. Roy. Meteor. Soc., 136, 2119-2131, 2010.

Peña, A., Floors, R. R., Sathe, A., Gryning, S.-E., Wagner, R., Courtney, M., Larsén, X. G., Hahmann, A. N., and Hasager, C. B.: Ten Years of Boundary-Layer and Wind-Power Meteorology at Høvsøre, Denmark, Bound.-Lay. Meteorol., 158, 1-26, https://doi.org/10.1007/s10546-015-0079-8, 2016.

Pope, S. B.: Turbulent Flows, Cambridge University Press, Cambridge, UK, 2000.

Raupach, M., Thom, A., and Edwards, I.: A Wind-Tunnel Study of Turbulent-Flow Close to Regularly Arrayed Rough Surfaces, Bound.-Lay. Meteorol., 18, 373-397, https://doi.org/10.1007/BF00119495, 1980.

Sathe, A., Mann, J., Barlas, T. K., Bierbooms, W., and van Bussel, G.: Influence of atmospheric stability on wind turbine loads, Wind Energy, 16, 1013-1032, 2013.

Savill, A. M.: Recent developments in rapid-distortion theory, Annu. Rev. Fluid Mech., 19, 531-575, 1987.

Sogachev, A. and Kelly, M.: On Displacement Height, from Classical to Practical Formulation: Stress, Turbulent Transport and Vorticity Considerations, Bound.-Lay. Meteorol., 158, 361-381, https://doi.org/10.1007/s10546-015-0093-x, 2016.

Sogachev, A., Cavar, D., Kelly, M., and Bechmann, A.: Effective roughness and displacement height over forested areas, via reduced-dimension CFD, Tech. Rep. DTU Wind Energy E-0161(EN), Risø Lab/Campus, Danish Technical University (DTU), Roskilde, Denmark, 2017.

von Kármán, T.: Progress in the Statistical Theory of Turbulence, P. Natl. Acad. Sci. USA, 34, 530-539, https://doi.org/10.1073/pnas.34.11.530, 1948.

Wyngaard, J. C.: Turbulence in the Atmosphere, Cambridge University Press, Cambridge, UK, 2010. 